\title{
Desarrollo de conocimientos estadísticos en futuros profesores de educación primaria a través de un proyecto de análisis de datos: posibilidades y limitaciones
}

\section{Developing statistical knowledge in prospective primary school teachers through a data analysis project: possibilities and limitations}

\author{
Hernán Rivas Catricheo ${ }^{1}$ \\ Juan D. Godino ${ }^{2}$ \\ Pedro Arteaga Cezón ${ }^{3}$
}

\begin{abstract}
Resumen: Este estudio describe los resultados de la aplicación de un proyecto de análisis de datos para desarrollar el conocimiento estadístico en futuros profesores de educación primaria. El proyecto fue aplicado a un curso de 70 estudiantes distribuidos en 16 grupos. A partir de los informes elaborados analizamos la competencia alcanzada, el tipo de respuestas y los errores manifestados. Los resultados obtenidos dejan en evidencia ciertos logros del enfoque constructivista aplicado, y también algunas limitaciones en los aprendizajes logrados. Se observa que si bien los participantes elaboran algunas respuestas con cierta autonomía, sus justificaciones están basadas en aspectos del conocimiento matemático-estadístico común del contenido, sin Ilegar a aplicar reflexiones más avanzadas, requeridas por el problema planteado. Esta situación sugiere que un enfoque didáctico de tipo constructivista debería ser complementado con momentos basados en la transmisión de
\end{abstract}

Fecha de recepción: 22 de agosto de 2017. Fecha de aceptación: 5 de junio de 2018.

1 Campus Villarrica. Pontificia Universidad Católica de Chile (Chile). hrivasa@uc.cl orcid.org/0000-00025847-0756.

2 Departamento de Didáctica de la Matemática. Facultad de Ciencias de la Educación, Universidad de Granada (España).jgodino@ugr.es orcid.org/0000-0001-8409-0258.

3 Departamento de Didáctica de la Matemática. Facultad de Ciencias de la Educación, Universidad de Granada (España).parteaga@ugr.es orcid.org/0000-0002-8347-7669. 
conocimientos, para superar situaciones de bloqueo generadas por la falta de conocimientos previos.

Palabras clave: formación de profesores; estadística; enseñanza constructivista; enseñanza por proyectos; conocimiento común y ampliado.

\begin{abstract}
This study describes the results of implementing a data analysis project to develop statistical knowledge in prospective primary school teachers. The project was applied to a group of 70 students distributed in 16 groups. From the reports prepared, we analyzed the competition achieved, the type of responses and the errors manifested. The analysis clearly shows certain achievements of the constructivist approach applied, and also some limitations on the learning levels. Although students prepare some answers with a degree of autonomy, their justifications are based on aspects of common statistical content knowledge without actually applying more advanced thinking, required by the problem posed. This suggests that the constructivist didactic approach should be complemented with moments of knowledge transmission to overcome blocking situations generated by the lack of previous knowledge.
\end{abstract}

Keywords: teacher education; statistics; constructivist teaching; teaching by projects; common and advanced knowledge.

\title{
1. INTRODUCCIÓN
}

El mayor énfasis dado a la estadística en la educación matemática ha generado nuevas exigencias en la formación de los profesores de educación primaria para enseñar la disciplina. No solo se requiere de una preparación adecuada sobre los contenidos a enseñar correspondientes a los niveles educativos en los que los docentes deben trabajar, sino también de un conocimiento "ampliado" sobre dichos contenidos, que les permita establecer conexiones con contenidos de niveles curriculares más avanzados.

La literatura sobre la dimensión instruccional de los procesos de formación de profesores en estadística es escasa y reciente. Sin embargo, existe una visión compartida en que el trabajo con proyectos de análisis de datos es una metodología didáctica apropiada para desarrollar la capacidad de producir e 
interpretar información estadística, favoreciendo distintos niveles de comprensión y competencia (Batanero y Díaz, 2011; Godino, Batanero, Roa y Wilhelmi, 2008). Esta metodología también es valorada en propuestas curriculares del ámbito escolar, donde se ha desarrollado un marco conceptual basado en la resolución de problemas de análisis de datos, como una forma de promover distintos niveles de alfabetización estadística (Franklin et al., 2005).

La metodología de enseñanza por proyectos es una forma de trabajo que apunta a contextualizar los contenidos a través de las diferentes fases de una investigación estadística: (1) Formular preguntas; (2) Recopilar datos; (3) Analizar los datos; (4) Interpretar los resultados. En cada una de estas fases se prevé que los estudiantes deben asumir un rol protagonista y aplicar los contenidos con cierta autonomía, lo cual promueve una enseñanza basada en un enfoque constructivista. Sin embargo, la puesta en funcionamiento de los conceptos, representaciones, procedimientos, argumentos y propiedades estadísticas involucradas en un proyecto de análisis de datos no resulta fácil, aun para los alumnos más aventajados.

En este estudio analizamos los resultados de la aplicación de un proyecto de análisis de datos enfocado en desarrollar aspectos del conocimiento estadístico común y ampliado (Godino, 2009), en futuros profesores de educación primaria. El marco metodológico de la investigación está situado en las investigaciones de diseño (Kelly, Lesh y Baek, 2008) o ingeniería didáctica (Artigue, 1989, 2011). Estas investigaciones pueden ser interpretadas como marcos metodológicos utilizados para estudiar los procesos de diseño y realización didáctica en espacios naturales de enseñanza. El diseño y la investigación se conciben como aspectos interdependientes, sobreentendiéndose que la investigación incluye no solo la fase de diseño, sino también la experimentación en contextos de clase y la evaluación de los resultados.

\section{MARCO TEÓRICO}

Para fundamentar el diseño y la realización didáctica en clase, aplicamos la idea de proyecto de análisis de datos. Este tipo de situaciones pueden ser interpretadas como problemas propios en la enseñanza de la estadística y se caracterizan por el planteamiento de preguntas que admiten respuestas basadas en datos que varían, en comparación con preguntas de tipo determinista. Los datos se generan en contextos particulares y son utilizados para responder preguntas 
relativas a dichos contextos. El análisis de los datos, inferencias y conclusiones requiere utilizar herramientas básicas de la estadística (tablas, gráficos, diagramas y estadísticos), que resultan necesarias para dar respuesta a las preguntas planteadas.

Con base en diferentes estudios sobre proyectos de análisis de datos (Batanero y Díaz, 2011; Burgess, 2008; Franklin et al., 2005) es posible distinguir cuatro fases en el ciclo de una investigación estadística:

- Formular preguntas: planteamiento de preguntas que conducen a respuestas no deterministas.

- Recopilar datos: recoger información mediante la aplicación de censos, encuestas por muestreo y realización de experimentos aleatorios.

- Analizar los datos: uso de estadísticos, tablas y gráficos para hacer comparaciones, describir una distribución o analizar la relación entre variables.

- Interpretar los resultados: elaboración de inferencias y conclusiones basadas en los datos obtenidos.

El trabajo mediante proyectos hace hincapié en estas cuatro fases del proceso de la investigación estadística y en el espíritu de una práctica estadística genuina, que promueva el uso del razonamiento estadístico para resolver problemas a través de los datos. Al respecto, Wild y Pfankuch (1999) proponen un modelo de razonamiento estadístico donde sintetizan los componentes fundamentales de este tipo de trabajo en los siguientes elementos:

- Reconocimiento de la necesidad de los datos: el reconocimiento de las carencias de las experiencias personales y la evidencia anecdótica lleva al deseo de basar las decisiones en la recaudación deliberada de datos.

- Transnumeración: la idea más importante en el aprendizaje de la estadística es la adquisición del proceso dinámico de cambio de las representaciones de los datos numéricos, para facilitar la compresión.

- Variación: el pensamiento estadístico moderno se refiere al aprendizaje y la toma de decisiones bajo incertidumbre, la cual surge de la omnipresente variación.

- Uso de un conjunto de modelos: la principal contribución de la estadística al pensamiento ha sido su propio conjunto de modelos específicos, esto es, marcos para pensar sobre determinados fenómenos que incluyen componentes aleatorios. 
- Conocimiento estadístico relacionado con el contexto: el material de base del pensamiento estadístico son el conocimiento estadístico, el conocimiento del contexto y la información contenida en los datos. El pensamiento en sí mismo es la síntesis de estos elementos para producir implicaciones, comprensiones y conjeturas.

Este modelo fundamenta la fase de diseño en nuestra investigación, particularmente la selección y secuenciación de tareas que se proponen en el proyecto. Efectuamos el análisis de los resultados con base en la noción de conocimiento matemático común y ampliado (conocimiento en el horizonte matemático) introducido en el modelo del MKT (Mathematical Knowledge for Teaching) (Hill, Ball y Schilling, 2008), interpretado desde el Enfoque Ontosemiótico del Conocimiento y la Instrucción Matemáticos (Godino, Batanero y Font, 2007). En este enfoque, el conocimiento común del contenido es entendido como el conocimiento compartido por el profesor con los estudiantes del nivel en el cual imparte la enseñanza y el conocimiento ampliado como el conocimiento compartido con los alumnos de etapas educativas posteriores (Pino-Fan y Godino, 2015).

\section{METODOLOGÍA}

Evaluamos los resultados de la implementación de un proyecto de análisis de datos que ha sido aplicado en el marco de un proceso de ingeniería didáctica basada en el Enfoque Ontosemiótico (Godino, Rivas, Arteaga, Lasa y Wilhelmi, 2014). Esta metodología propone tener en cuenta cuatro fases en el estudio de procesos de instrucción matemáticos: estudio preliminar, diseño de la trayectoria didáctica, implementación y análisis retrospectivo.

El proyecto de análisis de datos fue aplicado en una universidad de España a un grupo de 70 estudiantes para profesores de educación primaria, distribuidos en 16 equipos. Con el propósito de analizar los informes de trabajo, hemos definido tres tipos de variables:

- Grado de corrección: evaluación global de la competencia lograda, teniendo en cuenta aspectos del conocimiento común y ampliado; se asignan 2 puntos si la respuesta es correcta, 0 puntos si es incorrecta o no responde, y 1 punto si es parcialmente correcta. 
- Tipo de respuesta: clases de respuestas (procedimientos, justificaciones) que se ponen de manifiesto entre las respuestas correctas.

- Tipo de errores: errores que se manifiestan entre las respuestas erróneas y parcialmente correctas.

En la primera variable, los resultados de los informes son analizados desde el punto de vista cuantitativo y cualitativo. En las otras dos variables se hace un análisis cualitativo.

\section{DISEÑO DE LA TRAYECTORIA DIDÁCTICA}

Proponemos un proyecto de análisis de datos, a través del cual se pretende contextualizar y "motivar" la emergencia de las nociones y técnicas estadísticas descriptivas elementales (tablas de frecuencias, gráficos, promedios, dispersiones, comparación de distribuciones de frecuencias). El proyecto seleccionado, que describimos a continuación, es una versión adaptada del detallado en Batanero y Díaz (2011), considerando las variables y cuestiones que se indican:

\section{FORMULACIÓN DEL PROYECTO:}

Elaborar un perfil de los alumnos de la clase considerando las siguientes características (variables estadísticas): género (hombre, mujer); ¿haces deporte? (nada, poco, mucho); número de hermanos (incluyendo al propio estudiante, o sea, número de hijos en la familia); peso (kg); dinero que llevas en el bolsillo (cantidad de euros).

a. ¿Cuáles son las características de un estudiante típico o representativo de la clase? ¿Cómo de representativo es dicho estudiante respecto de la clase?

b. ¿Hay diferencias entre "chicos" y "chicas" en cada una de dichas características?

El proyecto fue implementado en dos sesiones de clases con trabajo en gran grupo (70 alumnos en la clase) y una sesión de trabajo práctico (donde la clase fue dividida en 3 grupos), utilizando la hoja Excel como recurso de cálculo. Se 
organizaron 16 equipos de trabajo con cuatro o cinco integrantes por equipo, quienes debían resolver el proyecto y entregar un informe final. Durante el desarrollo de las clases, el profesor actuó como "facilitador" de los aprendizajes, privilegiando el trabajo autónomo de los grupos, guiando las reflexiones y moderando instancias de debate.

\section{ANÁLISIS DE RESULTADOS}

Enseguida damos a conocer los resultados de los informes correspondientes a los 16 grupos teniendo en cuenta, frente a cada ítem, las cinco variables estadísticas consideradas en el proyecto (género, deporte, número de hermanos, peso y dinero que lleva en el bolsillo).

En la figura 1 mostramos un gráfico de cajas de la variable puntuación total obtenida por los estudiantes de este curso, teniendo en cuenta el grado de corrección de las respuestas a los distintos ítems del proyecto.

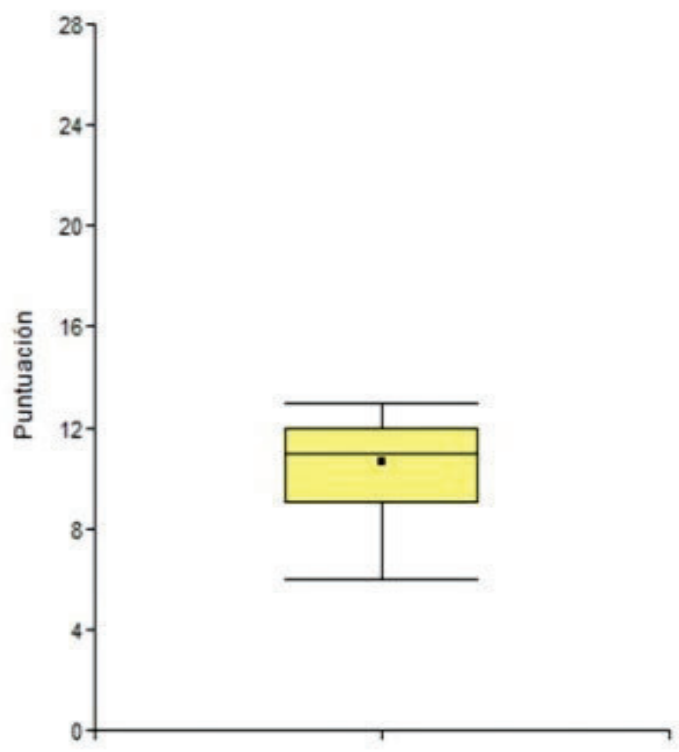

Figura 1. Gráfico de cajas de la puntuación total en el conjunto de ítem del proyecto. 
La puntuación máxima posible en esta evaluación es de 28 puntos. La media ha sido de 10.63; mediana, 11; cuartil inferior, 9.5; cuartil superior, 12; desviación típica, 1.82; mínimo 6 y máximo 13. Con base en estos datos, podemos afirmar que la tarea ha resultado difícil para los estudiantes.

A continuación analizamos los resultados de cada uno de los ítems.

\section{Ítem 1: ¿Cuáles son las características de un estudiante típico o representativo de la clase?}

En la tabla 1 presentamos los resultados para cada variable estadística, distinguiendo las respuestas correctas, parcialmente correctas y erróneas (y en blanco).

Tabla 1. Frecuencias y porcentajes de respuestas ítem $1(n=16)$.

\begin{tabular}{lcccccc}
\hline \multirow{2}{*}{ VARIABLE: } & \multicolumn{2}{c}{ Correctas } & \multicolumn{2}{c}{$\begin{array}{c}\text { Parcialmente } \\
\text { correctas }\end{array}$} & \multicolumn{2}{c}{$\begin{array}{c}\text { Erróneas } \\
\text { no responde }\end{array}$} \\
\cline { 2 - 8 } & Frec. & $\%$ & Frec. & $\%$ & Frec. & $\%$ \\
\hline 1. Género & 2 & 12.5 & 9 & 56.3 & 5 & 31.3 \\
2. Deporte & 8 & 50.0 & 8 & 50.0 & - & - \\
3. Número de hermanos & - & - & 13 & 81.3 & 3 & 18.8 \\
4. Peso & - & - & 14 & 87.5 & 2 & 12.5 \\
5. Dinero que lleva en el bolsillo & - & - & 14 & 87.5 & 2 & 12.5 \\
\hline
\end{tabular}

En la variable género (variable estadística cualitativa) se requiere resumir los datos en una tabla de frecuencias, e identificar al alumno típico a través de la moda. Esta información puede ser representada mediante gráficos. En los resultados obtenidos, los dos equipos que respondieron de forma correcta aplicaron la moda resumiendo los datos en una tabla de frecuencias; ocho de los nueve equipos que respondieron de forma parcialmente correcta reconocieron el estudiante típico sin aportar una justificación basada en el resumen estadístico de los datos (tabla de frecuencia o gráficos), y uno construyó correctamente una tabla de frecuencias sin responder la pregunta. El desarrollo de la pregunta frente 
a esta variable involucra aspectos del conocimiento común del contenido, el cual se manifiesta deficitario en 31,3\% de los equipos.

En la variable deporte la pregunta puede ser resuelta a través de la moda o la mediana. Esta variable es ordinal, si se ordenan los 60 valores de menor a mayor, se obtiene una serie del tipo: nada, nada,... (7 veces); poco, poco,... (44 veces); mucho, mucho,... (9 veces). El valor que ocupa la posición central de esta ordenación corresponde al valor poco, y coincide con la moda. Entre las respuestas obtenidas de los estudiantes, siete de los ocho equipos que respondieron de manera correcta aplicaron la moda construyendo una tabla de frecuencia y un gráfico de barras (un equipo incorporó además un gráfico circular), y uno solamente construyó la tabla de frecuencias. Los ocho equipos que respondieron de forma parcialmente correcta resumieron los datos en tablas de frecuencias y gráficos de barras, sin dar respuesta a la pregunta. Un error recurrente, manifestado en la construcción de gráficos, ha sido la falta de títulos y etiquetas en los ejes de los gráficos de barras. Ninguno de los equipos que construyó este tipo de gráficos incluyó dicha información.

Estos errores en la construcción de gráficos han sido detectados en investigaciones previas; por ejemplo, Arteaga, Batanero, Contreras y Cañadas (2016) evalúan los conocimientos que sobre gráficos estadísticos tiene una muestra de 207 futuros profesores de educación primaria al realizar un proyecto de análisis de datos. Aproximadamente $20 \%$ de los participantes cometieron errores relacionados con las escalas, etiquetas y rótulos de los ejes y título de los gráficos elaborados. Los autores mencionados resaltan la importancia de trabajar estos elementos de los gráficos con los futuros profesores, ya que son los que muestran la información del contexto de los gráficos, así como las unidades de medida utilizadas en la representación de la información; también destacan la importancia de ser cuidadosos con las escalas, pues la información de un gráfico puede distorsionarse a través de las mismas.

Los objetos matemático-estadísticos involucrados en esta pregunta son parte del conocimiento común del contenido. Si bien observamos un avance sustancial en el uso de tablas de frecuencias y gráficos con respecto a la pregunta anterior (impulsado tal vez por la mediación del profesor), también se manifiesta una impericia total sobre la posibilidad de aplicar la mediana.

En la variable número de hermanos (variable cuantitativa discreta) es necesario calcular estadísticos (media, mediana y dispersión) y construir gráficos para conocer la forma de distribución de los datos. En esta pregunta, de los 13 equipos que respondieron de manera parcialmente correcta, 10 calcularon la media 
interpretando equívocamente este valor; dos aplicaron el valor exacto de la media (2.75) y uno calculó la media sin dar respuesta a la pregunta. El uso de la media, en lugar de la mediana, muestra que si bien los estudiantes tienen un manejo apropiado del conocimiento común del contenido, no logran poner en funcionamiento aspectos del conocimiento ampliado. La puesta en práctica de este conocimiento supone tener en cuenta la forma asimétrica de la distribución y, en consecuencia, utilizar la mediana y no la media para responder la pregunta.

Algunos errores manifestados fueron el uso de la moda (valor especialmente indicado para variables cualitativas) y la aproximación de la media (2.75) a dos o tres hermanos. Este valor o el de la mediana (2.68), si hubiera sido aplicado, no tienen por qué coincidir con el valor que toma la variable en un sujeto particular. Finalmente, en la construcción de gráficos, un error que llama la atención es su construcción sin hacer un "resumen estadístico" de los datos (figura 2). En este caso se grafican los valores de la variable de acuerdo a la matriz de datos, en lugar de las frecuencias asociadas a cada valor. Al respecto Batanero, Arteaga y Ruiz (2010) definen cuatro niveles de complejidad semiótica en la construcción de los gráficos estadísticos por parte de futuros profesores de educación primaria; las representaciones como la mostrada en el Figura 1 corresponderían al segundo nivel, en el cual se representan todos los datos en el orden como fueron recogidos, sin llegar a formar una distribución de frecuencias. Los autores muestran que este tipo de representaciones dificulta la lectura e interpretación de los datos mostrados en los gráficos. Los autores recomiendan trabajar con gráficos de niveles de complejidad semiótica 3 o 4, en los que los futuros profesores ya llegan a la distribución de frecuencias de las variables estadísticas representadas.

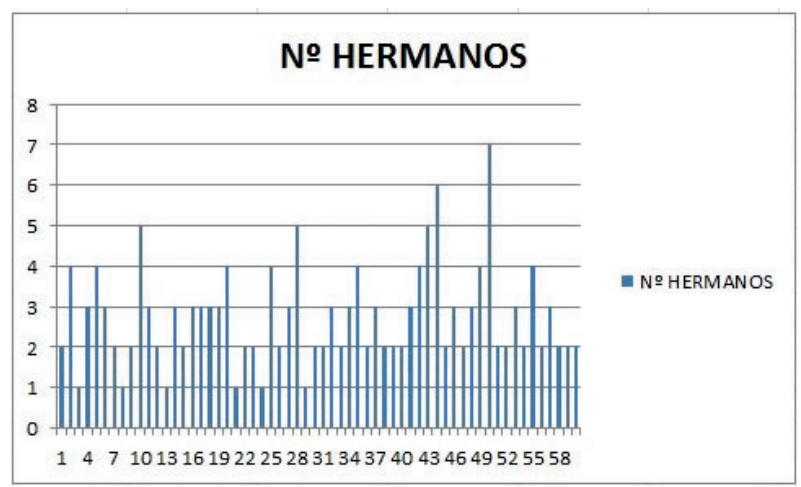

Figura 2. Gráfico "no estadístico". 
En la variable peso, al igual que en la variable anterior, deben calcularse promedios y dispersiones, y construir algún tipo de gráfico que permita ver la forma de distribución de los datos. La variable peso es cuantitativa y continua, por lo cual el resumen tabular de los datos debe hacerse mediante la agrupación en intervalos de clase. Entre las respuestas parcialmente correctas, nueve equipos calcularon la media interpretando equívocamente este valor, tres aplicaron el valor exacto de la media (61.45) y dos calcularon la media sin dar respuesta a la pregunta. Al igual que en la variable número de hermanos, ningún equipo utilizó la mediana, lo cual requiere interpretar adecuadamente la forma de distribución de los datos y la presencia de sujetos atípicos. En consecuencia, se manifiesta un bajo dominio del conocimiento ampliado del contenido estadístico.

Algunos errores manifestados fueron la determinación del alumno típico a partir del rango de mayor frecuencia absoluta (cálculo incorrecto de la moda) y la aproximación de la media (61.45) a 61 o 62 kg. En la construcción de gráficos, además de algunos grupos que no hicieron un resumen estadístico de los datos (uso de tablas de frecuencias), está presente la dificultad de reconocer el tipo de gráfico más apropiado para responder la pregunta. La figura 3 muestra la producción de uno de los grupos que optó por la construcción de un gráfico circular, el cual no permite visualizar la forma de la distribución ni distinguir la presencia de sujetos atípicos.

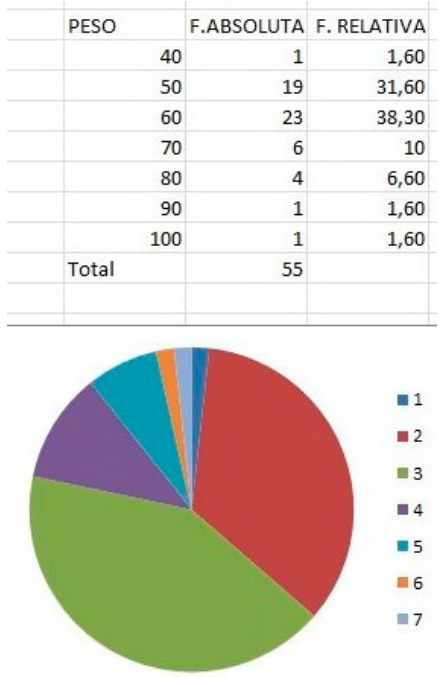

Figura 3. Selección inapropiada de gráficos. 
En la variable dinero se repite la necesidad de calcular promedios, dispersiones y construir gráficos para conocer la forma que presenta la distribución de los datos. Esta variable es vista como una variable cuantitativa continua y, por tanto, aplican los mismos procedimientos de la variable peso para la construcción de la tabla de frecuencias. En esta pregunta, entre las respuestas parcialmente correctas obtuvimos los siguientes tipos de contestación: ocho equipos calcularon la media interpretando equívocamente este valor, cinco aplicaron el valor exacto de la media (10.53) y un equipo calculó la media sin dar respuesta a la pregunta. Al igual que en las dos variables anteriores, ningún equipo utilizó la mediana (distribución asimétrica), lo cual confirma el escaso manejo del conocimiento ampliado del contenido estadístico.

Con respecto a los errores manifestados en esta pregunta, cuatro equipos aproximaron el valor de la media a 10 u 11, y dos han determinado el alumno típico a partir del rango de mayor frecuencia absoluta. Se repitieron los mismos errores de la variable anterior en la construcción de gráficos.

\section{Ítem 2: ¿Cómo de representativo es dicho estudiante respecto de la clase?}

La tabla 2 contiene los resultados de las respuestas dadas a este ítem para cada variable estadística, distinguiendo las respuestas correctas, parcialmente correctas y erróneas (y en blanco).

Tabla 2. Frecuencias y porcentajes de respuestas ítem $2(n=16)$.

\begin{tabular}{|c|c|c|c|c|c|c|}
\hline \multirow{2}{*}{ VARIABLE: } & \multicolumn{2}{|c|}{ Correctas } & \multicolumn{2}{|c|}{$\begin{array}{l}\text { Parcialmente } \\
\text { correctas }\end{array}$} & \multicolumn{2}{|c|}{$\begin{array}{c}\text { Erróneas } \\
\text { y no responde }\end{array}$} \\
\hline & Frec. & $\%$ & Frec. & $\%$ & Frec. & $\%$ \\
\hline 1. Género & 2 & 12.5 & & & 14 & 87.5 \\
\hline 2. Deporte & 2 & 12.5 & & & 14 & 87.5 \\
\hline 3. Número de hermanos & - & - & 14 & 87.5 & 2 & 12.5 \\
\hline 4. Peso & - & - & 14 & 87.5 & 2 & 12.5 \\
\hline 5. Dinero que lleva en el bolsillo & - & - & 14 & 87.5 & 2 & 12.5 \\
\hline
\end{tabular}

En las variables género y deporte, si bien no es posible determinar el grado de representatividad a través de los estadísticos obtenidos (moda, mediana), se espera 
que los estudiantes reflexionen sobre el porcentaje que representa la moda en cada caso. En la variable género 68\% (moda) de los participantes son mujeres y 32\% hombres; en la variable deporte 73\% (moda) hacen poco deporte.

Para las siguientes variables (número de hermanos, peso y dinero) esperamos que se afirme que si la medida de dispersión correspondiente es alta (respectivamente, baja), entonces el sujeto es poco (respectivamente, muy) representativo, ya que los datos estarían poco o muy agrupados alrededor del promedio. En estas tres variables los equipos que respondieron de forma parcialmente correcta elaboraron el cálculo de la desviación típica, pero no lograron interpretar dicho valor para responder la pregunta. El cálculo de la desviación típica supone un dominio avanzado del contenido matemático (su estudio se propone en los primeros niveles de la educación secundaria), que ha sido demostrado por la mayoría de los grupos (87.5\%).

\section{Ítem 3: ¿Hay diferencias entre chicos y chicas en cada una de dichas características?}

La tabla 3 presenta los resultados de las respuestas dadas a este ítem para las últimas cuatro variables estadísticas (en la variable género no es aplicable esta pregunta), distinguiendo las respuestas correctas, parcialmente correctas y erróneas (y en blanco).

Tabla 3. Frecuencias y porcentajes de respuestas ítem $3(n=16)$.

\begin{tabular}{lccccccc}
\hline \multirow{2}{*}{\multicolumn{1}{c}{ VARIABLE: }} & \multicolumn{2}{c}{ Correctas } & \multicolumn{2}{c}{$\begin{array}{c}\text { Parcialmente } \\
\text { correctas }\end{array}$} & \multicolumn{2}{c}{$\begin{array}{c}\text { Erróneas } \\
\text { y no responde }\end{array}$} \\
\cline { 2 - 8 } & Frec. & $\%$ & Frec. & $\%$ & Frec. & $\%$ \\
\hline 1. Deporte & 1 & 6.25 & 12 & 75 & 3 & 18.8 \\
2. Número de hermanos & - & - & 14 & 87.5 & 2 & 12.5 \\
3. Peso & - & - & 14 & 87.5 & 2 & 12.5 \\
4. Dinero que lleva en el bolsillo & - & - & - & - & 16 & 100 \\
\hline
\end{tabular}

Para responder a esta cuestión es necesario dividir el conjunto de datos en dos subconjuntos, correspondientes a los datos de los chicos y los de las chicas. Habrá que elaborar resúmenes tabulares, gráficos, calcular los estadísticos 
descriptivos para cada grupo y compararlos. Con las herramientas de la estadística descriptiva elemental solo es posible dar una respuesta aproximada y exploratoria. La determinación de la significación estadística de las diferencias observadas en las características de las distribuciones de frecuencias únicamente puede hacerse con métodos de estadística inferencial.

En la variable deporte, el equipo que respondió correctamente utilizó las frecuencias relativas, construyendo una tabla de frecuencias y un gráfico de barras adosadas. Entre los equipos que respondieron de forma parcialmente correcta, cinco construyeron tablas de frecuencias relativas y gráficos, interpretando equívocamente estos resúmenes estadísticos al responder la pregunta, y siete construyeron tablas de frecuencias relativas y gráficos sin dar respuesta a la pregunta (uno de estos equipos solamente construyó la tabla de frecuencias).

La interpretación a partir de las frecuencias relativas y del gráfico de barras adosadas supone un dominio del conocimiento ampliado del contenido, el cual quedó demostrado solamente en uno de los 16 informes recogidos.

Algunos errores manifestados en esta variable fueron la comparación de las dos submuestras (hombres y mujeres) mediante un gráfico de frecuencias absolutas (figura 4) y la comparación a través de la moda. Esto último conlleva a no reconocer las diferencias, ya que la moda es la misma en ambos grupos (poco deporte).

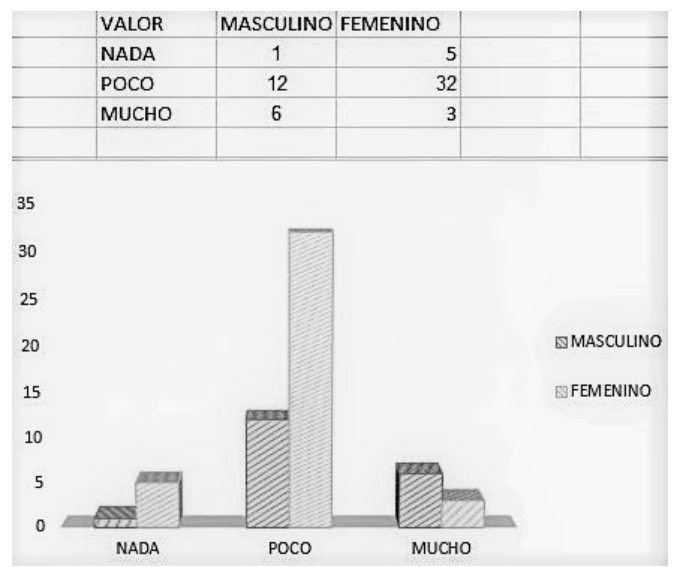

Figura 4. Comparación de muestras a través de frecuencia absolutas. 
En las variables número de hermanos y peso se obtuvieron respuestas iguales. Ningún equipo respondió correctamente la pregunta. Entre los equipos que respondieron de forma parcialmente correcta, la mayoría realizó bien el cálculo de la media y la mediana, pero aplicaron arbitrariamente la media para hacer la comparación (en ninguna de las dos variables emplearon representaciones gráficas para analizar la forma de distribución de los datos). El no tener en cuenta la forma de la distribución de los datos pone en evidencia un bajo manejo de este contenido, el cual es parte del conocimiento ampliado que los futuros profesores deberían dominar.

Uno de los errores manifestados en la variable número de hermanos fue la aproximación del valor de la media de los hombres (2.89) y de las mujeres (2.68) a tres, con lo cual se obtiene que no hay diferencias.

En la variable dinero los 16 equipos respondieron la pregunta comparando medias en lugar medianas, sin hacer ningún tipo de intento para analizar la forma que presenta la distribución. En este caso, a diferencia de las variables anteriores, las respuestas formuladas fueron erróneas, ya que al comparar las medias se obtiene una respuesta distinta de comparar las medianas: la media de los hombres (10.08) es menor que la media de las mujeres (10.73), y la mediana de los hombres (7) es mayor que la mediana de las mujeres (5).

\section{DISCUSIÓN FINAL}

El problema estadístico (proyecto de análisis de datos) que hemos propuesto a los estudiantes tiene un carácter abierto, ya que plantea cuestiones que pueden ser interpretadas de diversas maneras, sin sugerir la aplicación directa de una técnica estadística. Para responder las preguntas se requiere aplicar aspectos del conocimiento común y ampliado del contenido matemático, habiéndose intencionado un trabajo autónomo de tipo constructivista. La primera pregunta busca motivar el proceso de reducción de los datos estadísticos, identificando las variables, sus valores y frecuencias para construir la correspondiente distribución de frecuencias. Posteriormente requiere describir tal distribución mediante estadísticos de posición central, dispersión y forma para elegir un valor ideal que "represente" al conjunto de datos. La determinación de las diferencias estadísticas entre las dos submuestras (chicos y chicas) motiva la comparación de distribuciones de frecuencias y, por tanto, la indagación de la significatividad de las diferencias entre los promedios y dispersiones. Permite motivar, asimismo, la 
pertinencia de una comparación gráfica (por ejemplo, mediante diagramas adosados) de los pares de distribuciones.

El análisis de los datos ha mostrado que el cálculo de estadísticos (moda, media, mediana y dispersiones) y la construcción de gráficos de barras mediante la hoja de cálculo han sido logrados por la mayoría de los participantes. Sin embargo, aspectos más avanzados del razonamiento estadístico, como es el uso de la media o la mediana según la forma de distribución de los datos, la comparación de las dispersiones, la identificación de valores atípicos y su interpretación, así como la realización de histogramas de frecuencias representados sobre los mismos ejes cartesianos, han supuesto tareas con alto grado de dificultad para los estudiantes que han participado en esta acción formativa. Este resultado coincide con Peters (2009), quien afirma que muchos maestros son capaces de calcular la desviación típica e interpretarla, pero son incapaces de razonar sobre la desviación típica en conjunción con la media.

Por otra parte, se han presentado diversos errores que convendrá tener en cuenta en la implementación de nuevas propuestas. Algunos de estos errores fueron la aproximación de la media o la mediana sin tener en cuenta el contexto en que se aplican dichos valores, la construcción de gráficos sin hacer un resumen estadístico de los datos y la comparación de muestras a través de frecuencias absolutas.

Como conclusión de este estudio resaltamos que la enseñanza de las matemáticas, y en particular la estadística, debe partir y centrarse en el uso de situaciones-problemas (proyectos de análisis de datos) como una estrategia para dar sentido a las técnicas y teorías matemáticas. De esta manera, además, se hacen posibles los momentos exploratorios de la actividad matemática por parte de los estudiantes. Sin embargo, en la práctica matemática intervienen configuraciones de objetos matemáticos (conceptos, proposiciones, procedimientos, argumentos, representaciones) (Godino, Batanero y Font, 2007) que deben ser reconocidos por el formador para planificar su estudio. Tales objetos tienen que ser progresivamente dominados por los alumnos si se desea que progresen hacia sucesivos niveles avanzados de conocimiento, necesarios para una gestión idónea de la enseñanza. El estudio de tales objetos matemáticos y sus respectivos procesos asociados debe ser planificado por el formador al organizar los correspondientes momentos de validación, institucionalización y ejercitación para, de este modo, incrementar la idoneidad didáctica del proceso formativo. 


\section{Reconocimientos}

Trabajo realizado en el marco de los proyectos de investigación EDU2012-31869 (MINECO) y EDU2013- 41141-P (MINECO, España), y del Programa de Capital Humano Avanzado de la Comisión Nacional Científica y Tecnológica (CONICYT) de Chile.

\section{REFERENCIAS}

Arteaga, P., Batanero, C., Contreras, J. M. y Cañadas, G. (2016). Evaluación de errores en la construcción de gráficos estadísticos elementales por futuros profesores. Revista Latinoamericana de Investigación en Matemática Educativa, 19(1), 15-40.

Artigue M. (1989). Ingénierie didactique. Recherches en Didactique des Mathématiques, 9(3), 281-308.

Artigue M. (2011). L'ingénierie didactique comme thème d'étude. En C. Margolinas, M. Abboud-Blanchard, L. Bueno-Ravel, N. Douek, A. Fluckiger, P. Gibel, F. Vandebrouck y F. Wozniak (eds.), En amont et en aval des ingénieries didactiques (pp. 15-25). Grenoble: La Pensée Sauvage.

Batanero, C. y Díaz, C. (eds.). (2011). Estadística con proyectos. Departamento de Didáctica de la Matemática. Granada. Recuperado el 31 de julio de 2016 de http://www.ugr.es/local/batanero/publicaciones\%20index.htm.

Batanero, C., Arteaga, P. y Ruiz, B. (2010). Análisis de la complejidad semiótica de los gráficos producidos por futuros profesores de educación primaria en una tarea de comparación de dos variables estadísticas. Enseñanza de las Ciencias, 28(1), 141-154.

Burgess, T. (2008). Teacher knowledge for teaching statistics through investigations. En C. Batanero, G. Burrill, C. Reading y A. Rossman (eds.), Joint ICMI/IASE Study: Teaching Statistics in School Mathematics. Challenges for Teaching and Teacher Education. Proceedings of the ICMI Study 18 and 2008 IASE Round Table Conference. Recuperado el 5 de Agosto de 2016 de http://iase-web.org/documents/papers/rt2008/T3P1_ Godino.pdf.

Franklin, C., Kader, G., Mewborn, D., Moreno, J., Peck, R., Perry, M. y Scheaffer, R. (2005). Guidelines for assessment and instruction in statistics education (GAISE) report: A Pre-K-12 curriculum framework. Recuperado el 31 de julio de 2016 de http://www. amstat.org/Education/gaise/GAISEPreK-12_Full.pdf. 
Godino, J. D., Batanero, C., Roa, R. y Wilhelmi, M. R. (2008). Assessing and developing pedagogical content and statistical knowledge of primary school teachers through project work. En C. Batanero, G. Burrill, C. Reading y A. Rossman (eds.), Joint ICMI/ IASE Study: Teaching Statistics in School Mathematics. Challenges for Teaching and Teacher Education. Proceedings of the ICMI Study 18 and 2008 IASE Round Table Conference. Recuperado el 31 de Julio de 2016 de http://iase-web.org/documents/ papers/rt2008/T3P1_Godino.pdf.

Godino, J. D. (2009). Categorías de análisis de los conocimientos del profesor de matemáticas. UNIÓN, Revista Iberoamericana de Educación Matemática, 20, 13-31.

Godino, J. D., Batanero, C. y Font, V. (2007). The onto-semiotic approach to research in mathematics education. Zentralblatt für Didaktik der Mathematik, 39(1-2), 127-135.

Godino, J. D., Rivas, H., Arteaga, P., Lasa, A. y Wilhelmi, M. R. (2014). Ingeniería didáctica basada en el enfoque ontológico-semiótico del conocimiento y la instrucción matemáticos. Recherches en Didactique des Mathématiques, 34(2/3), 167-200.

Hill, H. C., Ball, D. L. y Schilling, S. G. (2008). Unpacking pedagogical content knowledge: Conceptualizing and measuring teachers' topic-specific knowledge of students. Journal for Research in Mathematics Education, 39(4), 372-400.

Kelly, A. E., Lesh, R. A. y Baek, J. Y. (eds.) (2008). Handbook of design research methods in education. Innovations in science, technology, engineering, and mathematics learning and teaching. New York, NY: Routledge.

Peters, S. A. (2009). Developing an understanding of variation: AP statistics teachers' perceptions and recollections of critical moments (Tesis de doctorado no publicada). The Pennsylvania State University. E.U.A.

Pino-Fan, L., Godino, J. D. (2015). Perspectiva ampliada del conocimiento didáctico-matemático del profesor. Paradigma, 36(1), 87-109.

Wild C. y Pfannkuch M. (1999). Statistical thinking in empirical enquiry. International Statistical Review, 67(3), 223-265.

HERNÁN RIVAS CATRICHEO

Dirección: Calle Bernardo O'Higgins \#501, C.P. 4930000 Villarrica, Chile.

Teléfono: (45) 2411830 